Wien klin Mag 2015 · 18:31

DOI 10.1007/s00740-015-0050-9

Online publiziert: 3. April 2015

๑) Springer-Verlag Wien 2015

\author{
V. Kienast \\ SpringerMedizin, Wien, Deutschland
}

\title{
Verführerisches Schweben
}

\section{Von der Relativität des Wissens}

Im Rückblick erscheint Vieles, was man persönlich erlebt, oder mitverfolgt hat, ganz anders. Neues Wissen kommt hin$\mathrm{zu}$, die eigene Erinnerung verblasst. Noch viel mehr verändert sich der Blick in die Vergangenheit, die man selbst nicht miterlebt hat - je weiter zurückliegend, desto weniger Informationen oder auch desto gefestigter sind die Einstellungen und Informationen dieser Zeit und ihren Ereignissen gegenüber. „Objektive“ Zeugen wie Schriftstücke, überlieferte Geschichten oder andere Fundstücke können immer nur ein Mosaik darstellen, das nie vollständig sein kann. Vielleicht lässt sich die Zeit mit wissenschaftlichen Methoden eruieren, lassen sich gewisse Konstellationen und Entscheidungen rekonstruieren. Die tatsächliche Lebenssituation, die Atmosphäre und Gefühle, Ängste und Freuden sind schwer aus dem Blick zurück nachzuvollziehen. Die Geschichte bleibt bruchstückhaft und immer beeinflusst. Der „Bias“ ist auf jeden Fall der zurückblickende Mensch.

\section{Wir wissen wie „sich alles ereignet hat"}

„Es stimmt natürlich immer noch“, schreibt der niederländische Autor Cees Nooteboom, „dass Geschichte nichts anderes ist, als eine Interpretation von Fakten und dass es stets auch eine Geschichte gibt, in der ein und dieselben Fakten sich anders ausnehmen." Und er nennt dafür ein einfaches Beispiel: „Ein Sieg oder eine Eroberung in dem einen Geschichtsbuch ist eine Niederlage oder eine Unterwerfung im anderen. Doch der Besitz von Fakten und außerdem die Kenntnis des Ausgangs, die zeitliche Distanz zu einem bestimmten Ereignis und das dadurch geringere Interesse eines späteren Betrachters vermögen eine fast göttlich Allmacht zu vermitteln, als könne man durch das Wissen wie ,alles sich ereignet hat', wie ein überlegenes Wesen über all diesen früheren Ereignissen schweben“. 1

Man wird natürlich nie wissen wie „sich alles ereignet hat" - wir wissen es auch in der eigenen Zeit nicht. Aber dieses allmächtig scheinende Schweben ist durchaus verführerisch - und hat natürlich seine Tücken. Der unreflektierte Glaube an die „Fakten“ macht nicht nur manipulierbar, sondern birgt auch ein nicht unbeträchtliches Risiko für den schmerzlichen Absturz. Hinterfragen und zweifeln kann die Flugbahn immer wieder korrigieren, auch wenn der eigene Glanz damit vielleicht ein bisschen weniger hell strahlt. Er wird voraussichtlich länger strahlen.

\section{Die unklare Bedeutung des vielen Wissens}

Entwaffnend offen hat Prof. Christoph Zielinski, einer der derzeit profiliertesten österreichischen Onkologen, kürzlich eine gewisse Ratlosigkeit in Bezug auf die onkologische Molekularforschung bekannt: Die Entschlüsselung des menschlichen Genoms, die vorab als Grundlage für die Heilung sämtlicher Krankheiten erhofft worden war, kann zwar heute von einem Techniker in einem Tag durchgeführt werden, Genmutationen können erkannt werden - aber was dies tatsächlich für den Patienten bedeutet, ist weiterhin unklar. „Wir haben geglaubt“, so Zielinski in einem Gespräch mit dem Genetiker Prof. Josef Penninger, „wenn wir ein mutiertes Gen finden, definieren und ein Target daraus machen, können wir die Krankheit heilen. Wir sind weit davon entfernt." Freilich wächst das Wissen permanent und exzessiv an, doch die Zusam-

\footnotetext{
1 Cees Nooteboom: Der Umweg nach Santiago, Frankfurt 1992.
}

menhänge und Auswirkungen müssen erst im Miteinander mit den unterschiedlichsten Disziplinen - und nicht nur der Medizin - zu einem umfassenderen Bild zusammengebaut werden, das eine höher Aussagekraft hat. „Wir haben enorm viel gelernt“, stellte Zielinski denn auch fest und: „Wissen wird wieder zu einem Privileg." Stets enthalten in diesem Wissen sollte auch das Bewusstsein für die Fehlstellen und die rasche Veränderlichkeit des Wissens sein

Meint Ihre

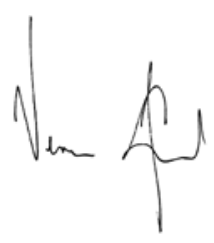

\section{Korrespondenzadresse}

\section{Kienast}

SpringerMedizin, Wien

Verena.kienast@springer.at 